\title{
Localizing Persons using Body Area Sensor Network
}

\author{
Cheng Guo, R. Venkatesha Prasad ${ }^{1}$, Jing Wang, Vijay Sathyanarayana Rao, Ignas \\ Niemegeers \\ Faculty of Electrical Engineering, Mathematics and Computer Science \\ Delft University of Technology, Mekelweg 4, 2600 GA Delft, the Netherlands
}

Keywords: Sensor Networks, Motes, Localization, RSSI, Triangulation

\begin{abstract}
Context awareness is an important aspect in many ICT applications. For example, in an intelligent home network location of the user enables session transfer, lighting and temperature control etc. In fact in a body area sensor network (BASN) location estimation of a user helps in realizing realtime monitoring of the person (especially those who require help) for better health supervision. In this chapter we first introduce many localization methods and algorithms from the literature in BASNs. We also present classification of these methods. Amongst them location estimation using signal strength is one of the foremost. In indoor environments, we found that the signal strength based localization methods are usually not accurate, since signal strength fluctuates. The fluctuation in signal strength is due to deficient antenna coverage and multi-path interference. Thus localization algorithms usually fail to achieve good accuracy. We propose to solve this problem by combining multiple receivers in a body area sensor network to estimate the location with a higher accuracy. This method mitigates the errors caused by antenna orientations and beam forming properties. We evaluate the performance of the solution with experiments. It is tested with both range-based and range-free localization algorithm that we developed. We show that with spatial diversity the localization accuracy is improved compared to using single receiver alone. Moreover, we observe that range-based algorithm has a better performance.
\end{abstract}

\section{BODY AREA SENSOR NETWORK AND LOCALIZATIONS}

With a number of devices equipped with sensors, micro-controllers and radio components, important parameters, such as body temperature, momentum, glucose levels, blood pressure or heart rate of the person can be monitored. In the meanwhile ambient intelligence (Ducatel, 2010; Aarts, 2003) is seen to be penetrating our daily lives. Being a way to realize ambient intelligence, the rapid development of these wireless sensor networks also extend to body area networks. Wireless sensors can be placed on persons, on cloths, etc. to monitor diverse vital health parameters of the persons. These devices form a Body Area Sensor Network (BASN). Such networks provide many novel applications in healthcare, fitness, and entertainment, which enable better quality of life of persons. A large amount of BASN applications are in healthcare domain. A BASN can help patients in hospitals gain more freedom. Wireless sensors can replace wired sensors monitoring patients. Thus they can move freely instead of being bonded to beds. More than in the hospitals, chronically ill patients or those are in recovery may return to their normal life at home with their physical conditions closely monitored by the doctors remotely. Another application of such body area sensor networks is helping senior citizens to manage their life in houses or even in public places without any support. In the above applications, context information has to be generated to provide control systems some form of context awareness. In case of emergency, data collected from sensors have to be collected and then processed in a control center to make a decision for actuation. Among the context

\footnotetext{
${ }^{1}$ Corresponding author
} 
information, location is an important one. No matter in hospital, home or in public area, locations of the patience have to be known so that first-aid can be provided timely. Moreover, the location information also provides doctors with the movement patterns of patients so that their living habits can be analyzed and used for disease prevention and diagnosis. Especially, people with Alzheimer disease should be monitored with wearable sensors and their house is equipped with other sensors for monitoring temperature, humidity, smoke or other hazardous gas and cameras for security surveillance. Some areas are dangerous such as kitchen where there are some sharp tools, gas stoves and electrical appliances. When the patient is moving in the house, sand moves into the kitchen his location should be known to the monitoring systems so that a video-camera in the kitchen is activated. An alarm and the video are sent to caretakers so that help can be provided timely in case required.

\section{APPLYING WIRELESS SENSOR NETWORKS FOR LOCALIZATIONS}

One of the most well-known localization systems is the Global Positioning System (GPS). However, due to poor penetration of the radio signals this system is not suitable for indoor localization (Savvides, 2001). A revolving technique in indoor environment is to use already deployed wireless sensor devices (in hospitals, buildings and other indoor area), together with wearable BASN to estimate the locations of the persons wearing them. This technique is highly practical and cost effective since all the sensors are already deployed and connected wirelessly for their usual tasks. Hence, localization does not add any additional investment on hardware infrastructure. There are a few requirements for reliable localization system. The localization system must have the features such as: (1) Accuracy: although many applications do not require localization to be accurate to centimeter, in many applications in home, buildings or hospitals, a monitoring system has to know where a user is, e.g. a living room or bed room. (2) Low complexity: in an indoor environment, location information is likely to be provided without dedicated hardware to avoid extra cost. Localization is expected to be an additional service on other devices, such as access points and environmental sensors. These devices work with embedded devices which can even be worn on the body. Computational and memory resource are limited on these devices. The localization algorithm or method must be as simple as possible and work inconspicuously to the users (Ducatel, 2010). (3) Real-time operation: location information has to be estimated in real time following the movement of the person. Therefore it has to be updated periodically at appropriate intervals. These intervals have to be decided by the speed of the users. Since we have the indoor environment as our user scenario, walking speed of 1 to $2 \mathrm{~m} / \mathrm{s}$ is considered as a moderate mobility in these scenarios. Therefore, locations shall be updated every a few seconds. In the literature, there are many approaches designed for different applications. Taxonomy for identifying location techniques in general was developed in (Hightower, 2001). The techniques are classified into Angle-of-Arrival (AoA), Time-of-Arrival (ToA), and the radio signal strength, which is frequently represented by Received Signal Strength Indicator (RSSI). Different techniques focus on different sensing data that is collected (Mutukrishnan, 2005). It has been shown that AoA and ToA methods are very accurate in ranging thus they estimate location with high accuracy. Especially when Ultra Wide Band (UWB) are combined with ToA method (Yu, 2004; Savvides, 2001) or both ToA and AoA (Deng, 2000), the accuracy can be improved even higher. A survey on UWB based localization methods is provided in (Gezici, 2005). Nevertheless, UWB requires extra hardware as reference points and all the devices have to have higher computational frequency so that the differences between ToA can be identified. Therefore it is not suitable for a BASN since the devices required by UWB methods are too large and consumes substantial power in wearable devices. Furthermore, the cost of a UWB system is much higher than that of a BASN. It is well known from many studies that the radio propagation is essentially related to the transmission distance. Given the transmitted signal power, RSSI is highly influenced by the distance. However, distance is not the only factor deciding RSSI. Both (Cox, 1984) and (Bernhardt, 1987) suggested that the distribution of receiving signal strength is a random and log normally distributed random variable with a distance dependent mean value. It is decided by a path loss exponent and a zero mean Gaussian distributed random variable accounting the effect of shadowing. Most of RSSI-based localization systems take advantage of existing indoor network 
devices such as WLAN or RFIDs (Jin, 2006). Along with the development of WSNs, RSSI-based methods use such a network more and more. The WSNs in most scenarios are implemented for monitoring environmental parameters such as temperature, humidity, presence, illumination etc. Together with a BASN, the two networks can cooperate to locate a person. A review of existing RSSI-based methods is provided in the next section.

\section{LOCALIZATION TECHNIQUES USING RSSI}

RSSI-based positioning algorithms are generally divided into two categories according to the classification in (Liu, 2004): range-based and range-free. The former makes use of the absolute distance or angle calibrated from the pre-measured RSSI map, which can be a set of "signatures" or RSSI to distance/angle relation. The latter, rather than using the information concerning the absolute distance, utilizes the geographic relationship between target motes and anchor motes.

RADAR (Bahl, 2000) and MoteTrack (Lorincz, 2005) are the examples of using the "signatures" for localization. Both protocols utilize a large set of pre-measured reference points on a map. Firstly the localization systems in the two protocols have a set of anchors. Then a mobile node is put at different points, which location is know, in the deployed area to measure the RSSIs to different anchors. The RSSIs are used latter for deciding a target mote's location. Afterwards the target node's RSSI to anchors are compared the neighboring reference points' RSSI to the same anchors. The closet reference points are selected. Location of these reference points form an area of which the gravity center is considered as the estimated location of the mobile node.

An enhanced RSSI-based localization system is proposed in (Lau, 2008). Although it is also range-based, it does not use "signatures". Instead, triangulation with Minimum Mean Square Error (MMSE) estimation is used to calculate the target node's location. The authors proposed a better ranging algorithm which assumes the target's current location is not far away from a previous one. Thus the two RSSIs should be highly correlated. So the current RSSI is averaged with the EWMA method.

APIT (He, 2003) is a good example of range-free localization protocol. The authors assume a lot of nodes are available in the area where the localization is needed. Nodes can exchange their RSSIs observations of three anchors. The three anchors form a triangle. If a node in the target node's neighborhood has RSSIs to the anchors larger than the target node's, then the target node must be outside the triangle formed by the anchors. Otherwise it is inside. Changing another three anchors, another triangle can be formed. The whole localization area is divided into small areas; once a triangle is formed the possible areas where the target node may be are given more weight. After all the combination of anchors is tried, the highest weighted areas' gravity center is estimated as the target node's location. ROCRSSI (Liu, 2004) is similar to APIT. However, the rings are used to replace triangles. The RSSI $p$ from the target to an anchor is compared to the RSSIs from the anchor to other anchors. We can always find a pair RSSIs between anchors that $p$ is larger than one is smaller than the other one. Then a ring can be drawn that the target node is possibly inside. Again several rings add weights to the possible small areas the target node may reside. The estimation location is the gravity center of the highest weight area.

Received Signal Strength Indicator (RSSI) is widely exploited for localization in the literature. However, because of the small size, wearable characteristics and cost restrictions, health care wireless sensor devices normally use an on-chip antenna which in turn has no perfect omnidirectional beam properties. As a consequence, the antenna orientation of the devices has a large influence on the received signal strength. Factors such as complex indoor radio propagation and the movement of people usually cause RSSI-based localization algorithms to fail to achieve a good accuracy. In this chapter, we propose a method, which takes advantage of multiple wireless devices in a BASN to improve the accuracy of estimates of location of a person in an indoor environment. The highlights of this method are: (a) nullifying the effects of antenna orientation by using multiple devices; (b) using the spatial redundancy in the estimations; and (c) implementation of the system to show our method performs better under similar circumstances. 


\section{LOCALIZATION WITH MULTIPLE DEVICES}

Range-based localization algorithms usually fail to achieve good accuracy due to deficient antenna embedded, fading, shadowing, and people movements. In this chapter, we try to solve the first two problems by combining multiple wireless devices in a BASN. Their diverse antenna orientations and small distance can be exploited to increase the localization accuracy. Moreover, it was shown in the literature that the relation between RSSI and range is not one to one. Transceivers apart of different distances, sometimes up to 10s of meters, can receive packets with the same RSSI (Aguayo, 2004; Zhao, 2003). Therefore, absolute distance estimation is tricky.

Although range-free algorithms avoid using absolute distance estimation between anchor and target nodes, it has to use relative distances between anchors. If the antennas on the anchors also have the problem on orientation, the relative positions of anchors are not precise anymore thus the location estimated is not accurate. We will combine the multiple-devices-solution with representatives from both categories to test how much this solution can improve performance of the protocols.

In the following, we firstly introduce the advantages of using multiple devices for localization. For completeness, we introduce the two representatives from range-based and range-free algorithms that we proposed in (An, 2006; Wang, 2006).

\section{Localization with Multiple Devices in a BASN}

Small BASN devices normally transmit with on-chip antennas. These antennas' transmission pattern is far from perfect omnidirectional (Tmote sky datasheet, 2006). We verified the deficient coverage of the antenna by putting the transmitting and receiving motes $4 \mathrm{~m}$ apart within the line-of-sight (An, 2006). We measured the RSSI values at 8 different antenna directions in steps of $45^{\circ}$. In Fig. 1, we show that the antenna has the strongest strength of $-50 \mathrm{dBm}$ at $0^{\circ}$ and the smallest signal strength of about $-65 \mathrm{dBm}$ at $90^{\circ}$. We observed that RSSI varies in a range of around $15 \mathrm{dBm}$ for the static case.

This problem can be solved by continuously rotating the antenna direction and taking the average of the RSSI values received from different directions. However, this method is neither agile nor feasible. Since there may be many wireless sensor devices in a BASN, we can actually use the RSSI from different devices to calculate the location with the average RSSI. Or we can let the devices calculate the location independently and take the average of the calculated locations. Both ways may mitigate the estimation error induced by the deficient antenna radiation pattern. We will show the performance of both methods in Section V.

Using multiple devices can also reduce the estimation error caused by fading, since the signal is transferred in different paths. We can use the so-called micro diversity, which refers to that the antennas are at a distance of an order of a wavelength, to combat fading. 


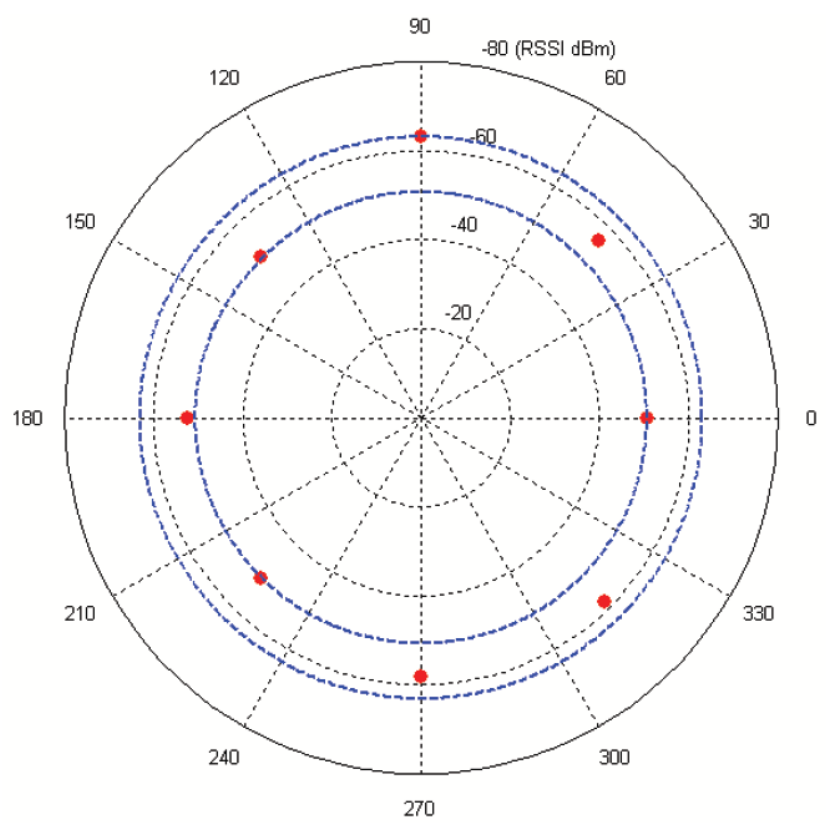

Figure 1. Antenna Orientation Effect (An, 2006)

\section{Range-based Localization Algorithm}

We proposed an enhanced triangulation algorithm in (An, 2006). The optimization is that we give anchors which have higher RSSI to a target higher weight so that they are trusted more and used firstly to calculated a location. The next question is how large weight we shall assign to an anchor. Since we do not know the reliability of any measure RSSI it is hard to assign the weight. We proposed to use the following way. We firstly have to plot out an empirical curve showing relation between RSSI and distance, as shown in Fig. 2. All the distances were estimated by this relation with received RSSIs. We can see from Fig. 2 that the higher the RSSI the steep of the curve is. We use the slopes of the curve as the weight of an anchor. To be specific, we record all the RSSIs received from anchor $i$ and mapped them to the empirical relation curve. For each of them we have a slope of the mapped segment, i.e. if we have a RSSI of $-68 \mathrm{dBm}$ then we can get the slope of segment 4 meter to 6 meter. We get the weight of the anchor when it receives the $j$ RSSI as

$$
W_{i}=\frac{\text { Slope }_{i}^{j}}{\max \left(\text { Slope }_{i}^{j}\right)}
$$

Then we try to find the position of the target mote by minimizing

$$
\Delta=\left(\sum_{i=1}^{N} W_{i} \Delta_{i}^{2}\right)^{1 / 2}
$$

Where $\Delta_{i}$ is the error calculated by $f_{i}\left(x_{e}, y_{e}\right)=\left|d_{i}-\sqrt{\left(x_{i}-x_{e}\right)^{2}+\left(y_{i}-y_{e}\right)^{2}}\right|$. In the last equation, $d_{i}$ is the estimated distance between the target mote and the anchor mote $i$, which is located at $\left(x_{i} ; y_{i}\right)$.For more details about the algorithm, please refer to (An, 2006).

\section{Range-Free Localization Algorithm}

Range-free localization algorithms utilize the relative position to decide the possible region where the target mote may reside (Wang, 2006). Take for example, Fig. 3, Anchor $A$ receives packets from Anchor $B$ and estimates RSSI, so as Anchor $C$, and Target $T$. Thus we may expect the relationship RSSIAB > RSSIAT > RSSIAC. Therefore, we can conclude that the Target $T$ is most likely to be in the grey ring. We 
can derive a series of such grey areas so that the estimated position is decided as the gravity center of the final intersectional area.

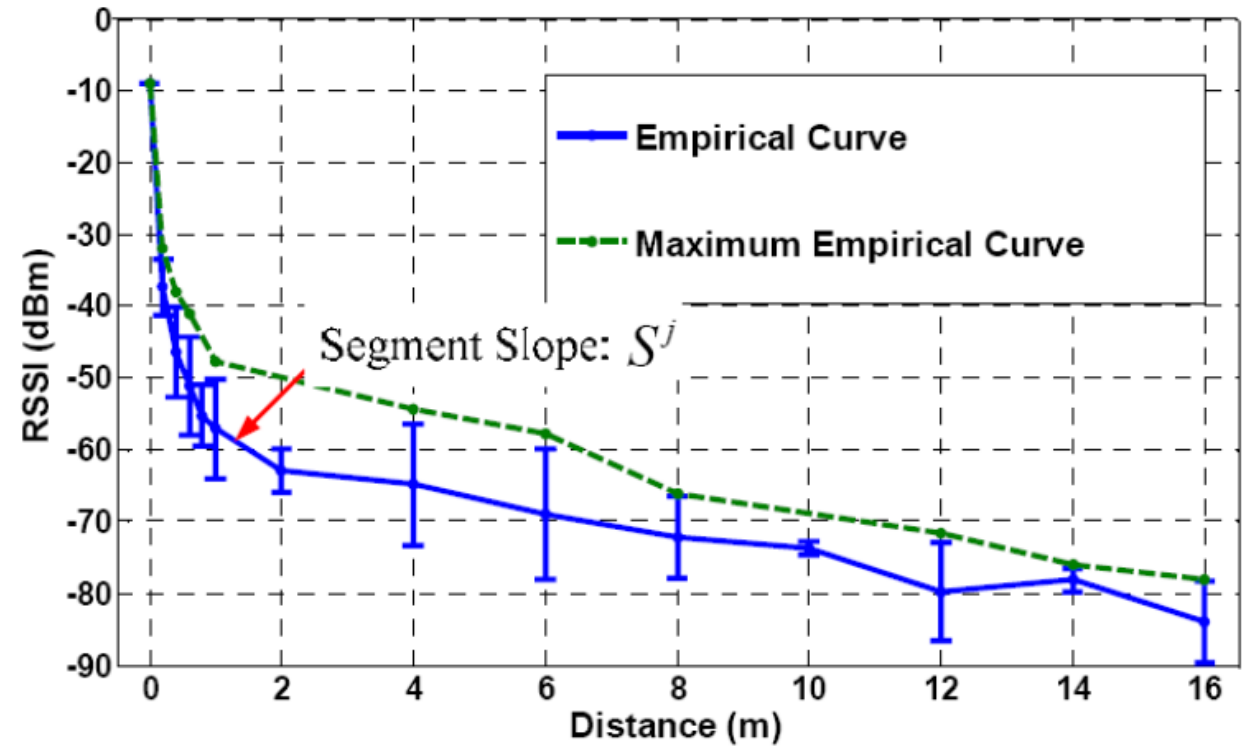

Figure. 2. Empirical Relation Curve (An, 2006)

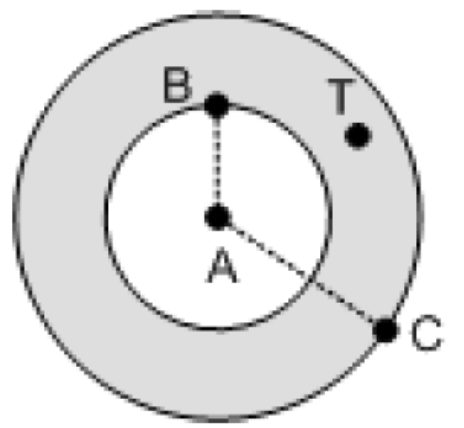

Figure. 3. An Illustration of Range-free Localization Algorithm (Wang, 2006)

We extend this basic algorithm by ensuring more credibility to the measured RSSI. The same theory as the range-based algorithm is used. If the distance between transceivers is small, the RSSI rapidly changes with a tiny increment or decrement of the distance. Contrarily, a large change of distance may only lead to an imperceptible change of RSSI if the distance is large. Therefore, we assign lower credibility if the RSSI has lesser value which corresponds to a bigger estimation error, i.e., a longer distance. In our algorithm, each anchor node has a neighbor list sorted in descending order based on the received RSSI. We briefly explain the algorithm with an example. In Table I, Anchor 6 decides that Target $T$ is in the ring between Anchor 5 and Anchor 7. However, if $d_{65}>d_{67}$, the ring cannot be generated. Therefore, we refer to Target $T$ 's neighbor list. Target $T$ also has a sorted neighbor list based on the RSSI from the anchor nodes, which is also shown in Table I. In Target T's list, the RSSI from Anchor 5 is higher than the RSSI from Anchor 7. Thus, we envisage that RSSI from Anchor 5 has a higher reliability than the RSSI from Anchor 7. Therefore, we ignore Anchor 7 and move to Anchor 3. If Anchors 5 and 3 can generate a ring, we proceed to the next step. If Anchor 5 and 3 still cannot generate a ring, we go back to trust Anchor 7 and ignore Anchor 5. If Anchor 2 and 7 can generate a ring, then algorithm proceeds further. The accuracy of the process is based on the assumption that, the majority of RSSI are reliable. Although RSSI is disturbed by a multitude of factors, the above assumption is usually true in most of the situations when averaged over a small duration. 
After generating a series of rings, we count the number of times an area is covered. Every time a ring covers an area, the counter of the area increases by one. Then, we calculate the gravity center of the area to find the location. However, since we already know that higher RSSIs have higher credibility, we give overlapping areas with a higher RSSI more weight. If an area is covered by a ring, the increase of the area counter is related with the reliability weight of the ring. For Anchor $I$, the reliability weight is defined as:

$$
w_{T I}=\left(\frac{1}{R S S I_{T I}}\right)^{n}
$$

where, $R S S I_{T I}$ is the RSSI received by Anchor $I$ from Target $T$ and $n$ is the exponential index of the radio propagation model. Now we use the refined RSSI conceived from multiple sensors (acting as a bunch of targets) into the above expression to get a better accuracy.

\begin{tabular}{|l|l|l|l|}
\hline \multicolumn{2}{|c|}{ Neighbor list of Target (ID 1) } & \multicolumn{2}{c|}{ Neighbor list of Target (ID 6) } \\
\hline Anchor ID & RSSI (dBm) & Node ID & RSSI (dBm) \\
\hline 2 & -54.387 & 4 & -64.7273 \\
\hline 3 & -83.801 & 8 & -66.3333 \\
\hline 4 & -73.778 & 2 & -76.4667 \\
\hline 5 & -65.529 & 5 & -78.5714 \\
\hline 6 & -80.029 & 1 & -80.0294 \\
\hline 7 & -82.002 & 7 & -82.7143 \\
\hline 8 & -85.791 & 3 & -88.6316 \\
\hline
\end{tabular}

Table 1.Example of selecting reliable RSSI

\section{EXPERIMENTAL SETUP AND RESULTS \\ Setup}

We conducted a series of experiments at the $19^{\text {th }}$ floor of our faculty. The devices used in the experiments were TMote-Sky sensors with 2.4 GHz IEEE 802.15.4 compliant Texas Instruments CC2420 transceivers (CC2420 RF transceiver datasheet, 2008). The transceiver provides RSSI in a range of $100 \mathrm{dBm}$ for every received packet, which is the received strength signal reading averaged over eight symbol periods. IEEE 802.15.4 (Gutierrez, 2001) uses 2.4 GHz DSSS RF modulation with a data rate of $250 \mathrm{kbps}$. In our experiment, the IEEE 802.15.4 packet header and payload of 17 bytes and 9 bytes were used resulting in 26 bytes packet.

We bound four motes oriented in four directions on a $30 \mathrm{~cm}$ high platform made of polystyrene. The distance between any two motes was about $6 \mathrm{~cm}$, which is half the wavelength of $2.4 \mathrm{GHz}$ radio. We expected that distance may help us to combat the effects of fading. The four motes were connected to a PC via USB cables. Thus, the received packets were directly relayed to the PC, which acts as the sink of the BASN. The environment was checked to be Wi-Fi free. All the experiments were carried out during weekends minimizing the effect of human movements and other activities.

We deployed in total 11 anchors on a straight line in the middle of the corridor. All the anchors were also lifted with the $30 \mathrm{~cm}$ high polystyrene. The distance between any two anchors was $4 \mathrm{~m}$. All the anchors were powered by external DC supply to avoid the fluctuation in power supply due to different battery levels. The arrangement of the motes is shown in Fig. 4. The blue nodes in the figure are anchors and the red nodes represent the positions of target nodes. The horizontal distances between the anchors and the targets are $2 \mathrm{~m}$. We tested 10 target positions in line with the anchor nodes, called Scenario-A and another 9 positions which were close to the wall, called Scenario-B. With Scenario-B, we want to check the influence of reflections from the wall on the accuracy.

In each target location, we run an experiment for 60s. During each experiment, the anchors randomly sent a beacon message every second and the target randomly selected one in each $1 \mathrm{~s}$ interval. Hence, for each measurement, 60 beacon messages were sent by every anchor mote. Meanwhile, the target motes reported the received packets to the sink and the anchors recorded the beacons they received into their local memory. Afterwards, one of the target motes polled all the anchors of the recorded data. For each 
received beacon, sender, receiver, packet identifier, and RSSI were collected and gathered at the PC for further computations and analysis. Then, all the motes were reset and we moved the target motes to the next location.

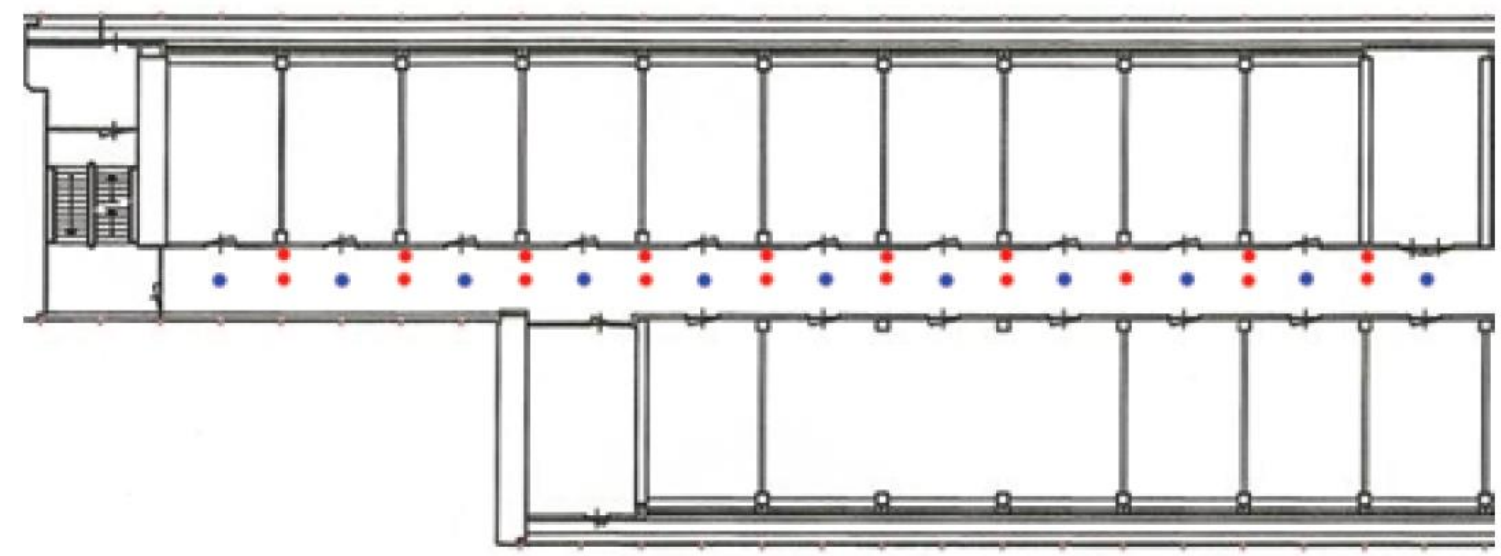

Figure 4. Location of anchors and Targets

\section{RESULTS}

We will show in this section the performance of using multiple devices for localization. Both range-based and range-free algorithms are tested. We take the averaged RSSI from the target motes to estimate a location. Then the estimation error is compared with the error using the RSSI from each target and the maximum RSSI among all target nodes. The joint performance of using multiple devices with rangebased is compared to that with range-free algorithm to select a winner. We are also curious about whether averaging RSSIs collected from a longer period may improve localization accuracy. Furthermore, instead of averaging RSSIs from multiple devices, we can also take the average of estimated positions from individual target device or the gravity center of the estimated positions. We will show which way may improve the localization accuracy the most.

Considering the requirements of a real-time indoor person tracking system, it is then important that location updates are done frequently. Hence, for a moving person, we must typically not use more than 5 samples. Therefore, we firstly use 5 samples for estimation. Fig. 5 shows the estimation result with rangefree algorithm. It compares the sample RSSI averaged on four target motes and the RSSI from each target mote. Fig. 8 shows the case with range-based algorithm. Please note that in the two figures, the scale of estimation error is different. We can see that in both cases, using the averaged RSSI still gives best estimation with an largest error about $3 \mathrm{~m}$, which is indicated by the thick red line in the figures. The range-based algorithm performs better in $90 \%$ positions where errors are less than half meter. The only exception happens in one location where the error is only a bit large than $3 \mathrm{~m}$.

Secondly, all the 60 beacons collected by each target mote were used for the estimation. The application scenario can be that a person stands in a location for some time. We want to see whether averaged RSSI from a longer period may reduce the estimation error. Again Fig. 7 shows the estimation result with range-free algorithm and Fig. 7 shows the case with range-based algorithm. In general, using the average still gives higher accuracy. Compared to the 5 samples case, all the estimation errors are below $2 \mathrm{~m}$. It means that averaging RSSIs from longer period indeed removes some jittering in the RSSI values.

However, the case is not always true. In the added 55 samples, some may add larger errors. Therefore $90 \%$ of estimation errors are smaller than half meters in the 5 samples case, but only $40 \%$ are smaller than haft meter in the 60 samples case. This time a large exception in estimation error happens with range-free algorithm. Since the complex nature of radio propagation and localization algorithms, it is hard to explain why the exception happens sometimes with range-based algorithm, but happens with range-free algorithm 
the other times. A possible reason may be that the accuracy of range-free algorithm does not depend on the magnitude of RSSI deviation. As long as rings can be formed, even a large RSSI deviation may be ruled out by other rings. But in some cases, even a small deviation may cause a serious error since rings can not be formed anymore or formed wrongly. Compared to range-free algorithm, the accuracy of rangebased algorithm depends on the RSSI deviation. Averaging RSSIs from longer period reduced the deviation hence large estimation errors are removed.

For Scenario-B, the estimation results when using 60 beacons is drawn in Fig. 9. In general, the average method outperforms and provides better accuracy than using an individual target mote. However, due to extra reflections and shadowing, the median error is around $2 \mathrm{~m}$ and in the $90 \%$ of the cases the errors are below $6 \mathrm{~m}$.

We are also interested in the comparisons in estimations between 5 beacons case ( $4 \mathrm{~s}$ to $8 \mathrm{~s}$ ) and 60 beacons case. In Fig.10, which is plotted with data from Scenario-A, we can see that the estimation accuracy is generally the same except for the exception introduced in Fig. 9.

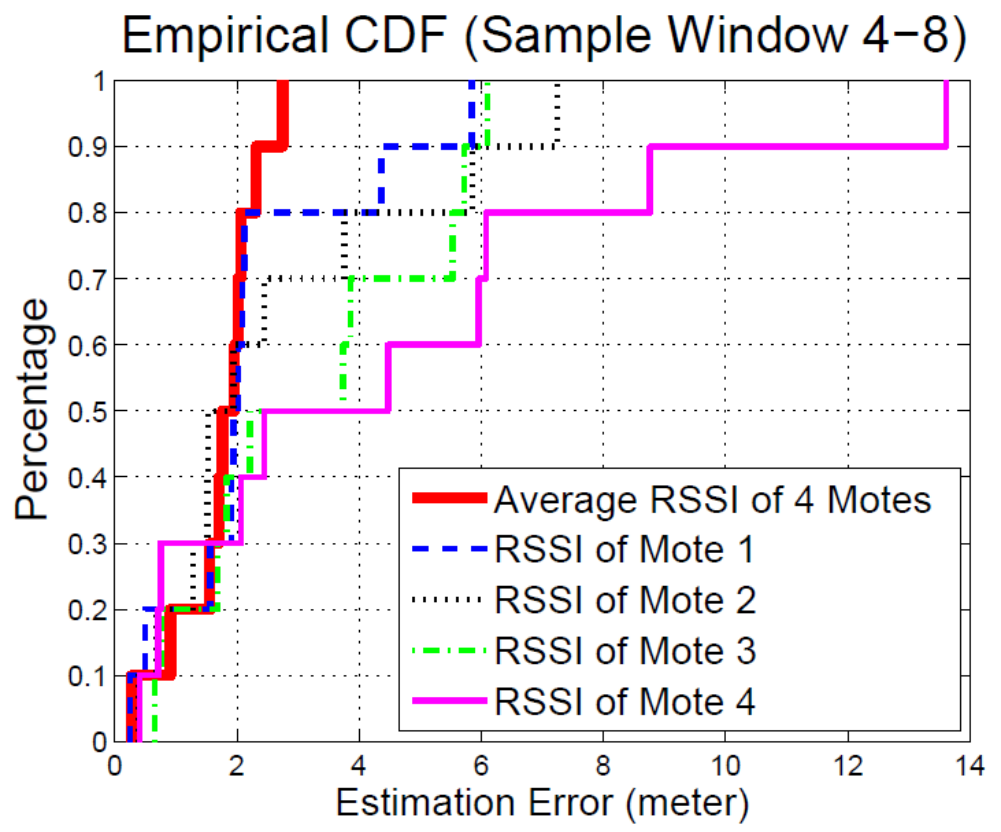

Figure. 5. Estimation error comparison with Range-free algorithm in Scenario-A: 5 samples each target 


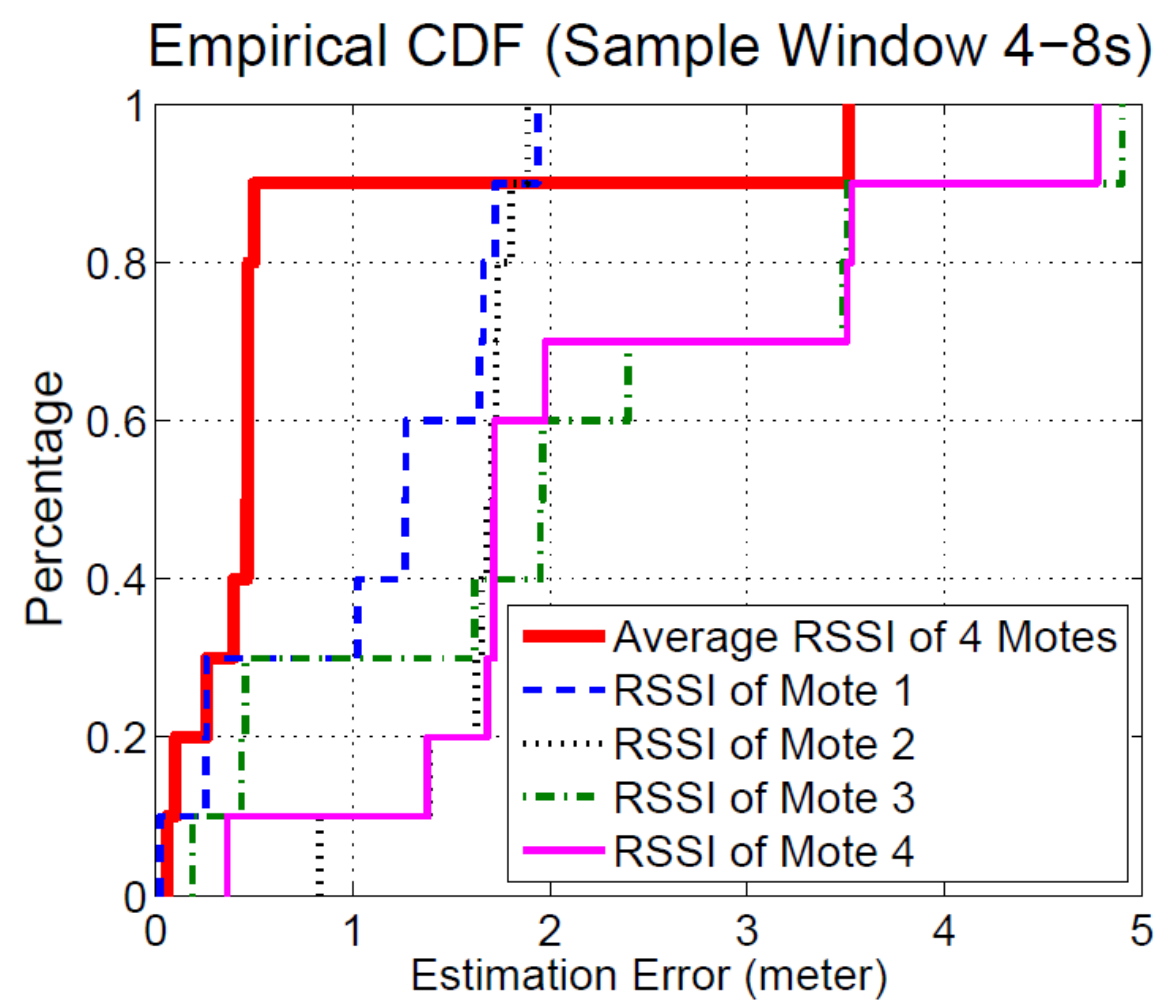

Figure 6. Estimation error comparison with range-based algorithm in Scenario-A: 5 samples each target

Another method to utilize the RSSI from multiple devices is to estimate the location of each individual targets and then calculate the average location. In Fig. 10, we investigate the accuracy of this method. However, we can see that the average RSSI method outperforms the average position method in general.

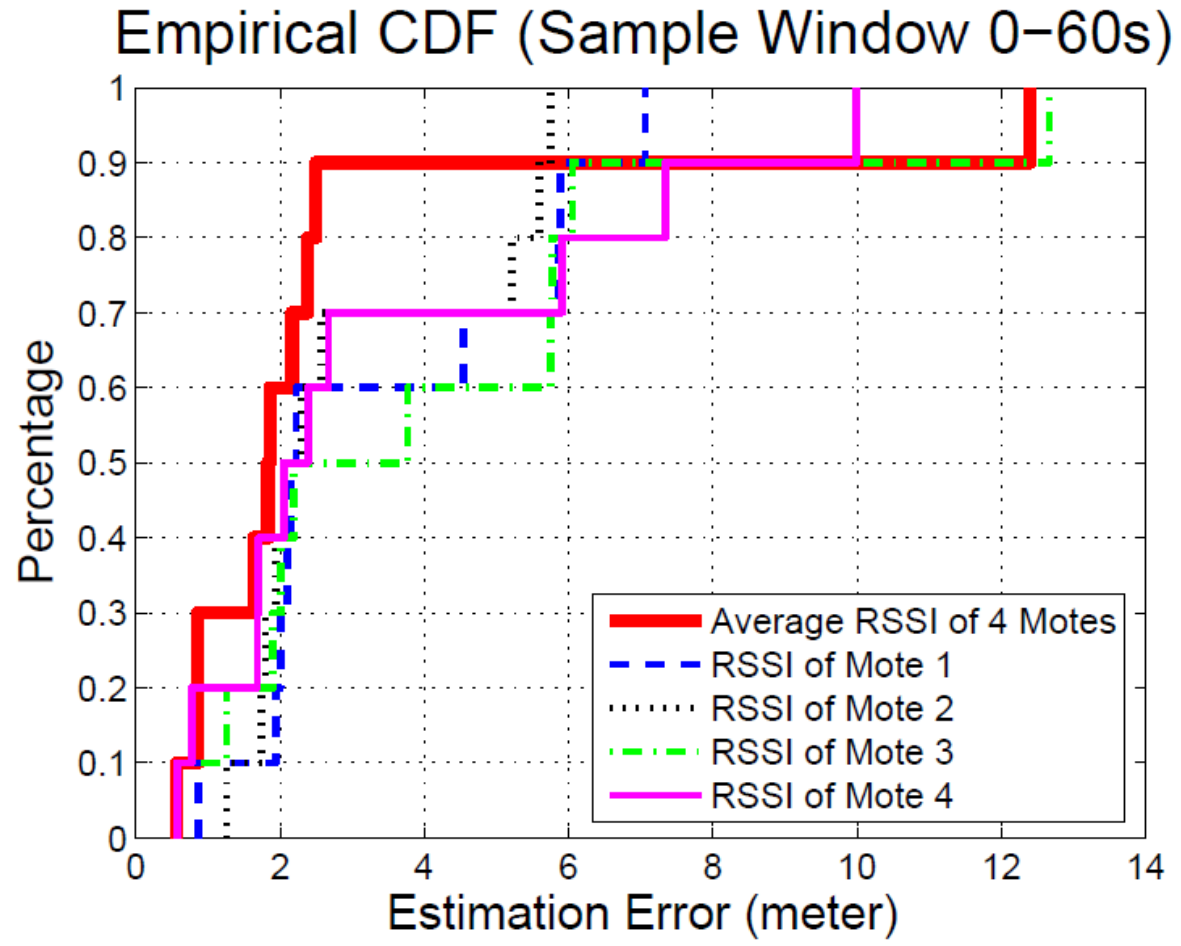

Figure 7. Estimation error comparison with Range-free algorithm in Scenario-A: 60 samples each target 
This is due to the fact that an individual measurement going haywire can pull the location farther away since slight change in RSSI contributes to higher distance when the target is away. Thus it is better to use averaged RSSI than averaged estimated location.

For the estimation using a small number of beacons, we also examined the estimation stability on the time scale. We divided the whole 60s data into 12 estimations of 5s each. The 10 locations in Scenario-A and the 9 locations in Scenario-B are all plotted in Fig. 11 with both algorithms. The curves show the average estimation error of the 12 estimations and the bars show the standard deviation. We can see that the stability of range-based algorithm is better than that of range-free algorithm. Most of the estimation errors of range-based algorithm in different locations are less than $2 \mathrm{~m}$. The estimation variations in most locations are so small that are hardly observed. Even the estimation errors in Scenario-B are small. With range-free algorithm, the largest error is more than $5.5 \mathrm{~m}$ in Scenario-A. Due to the structure of the floor, some places have more obstacles than others and thus suffer more from shadowing effect, which degrades the estimation accuracy. The estimation errors also vary with time. Interestingly, the locations with larger error also have larger deviations due to the complex structures around the location. In Scenario-B, the average error is a little less than $7 \mathrm{~m}$ and the standard deviation is more than $4.5 \mathrm{~m}$.

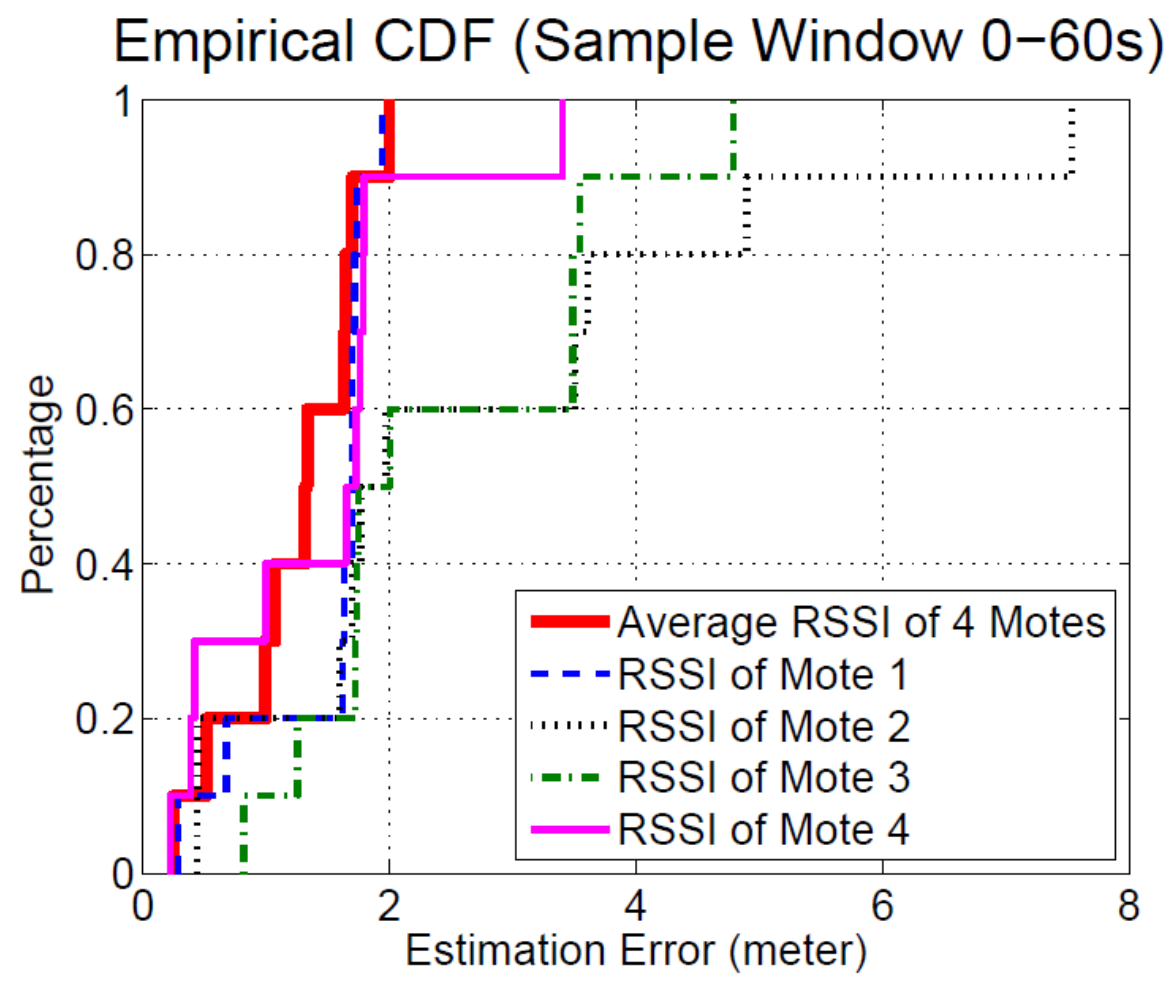

Figure 8. Estimation error comparison with range-based algorithm in Scenario-A: 60 samples each target 


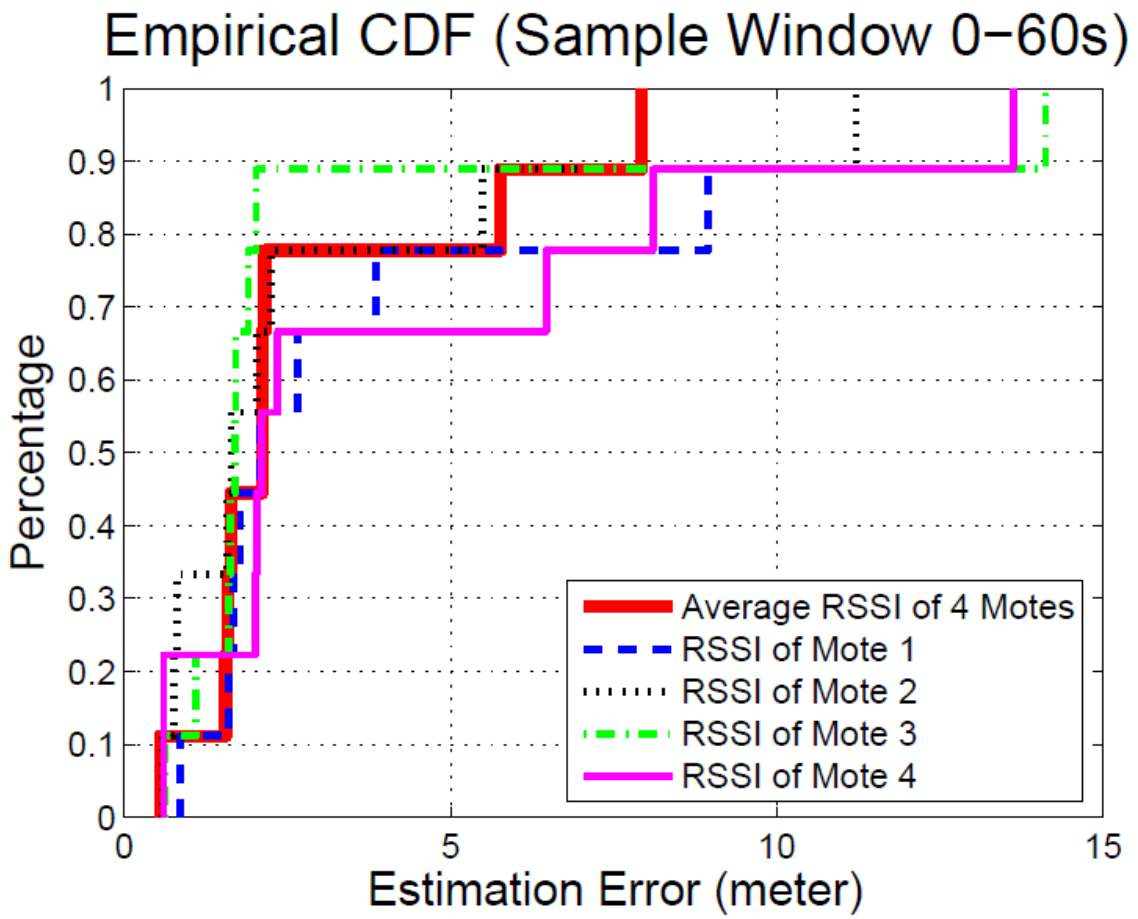

Figure 9. Estimation error comparison with range-free algorithm in Scenario-B: 60 samples each target

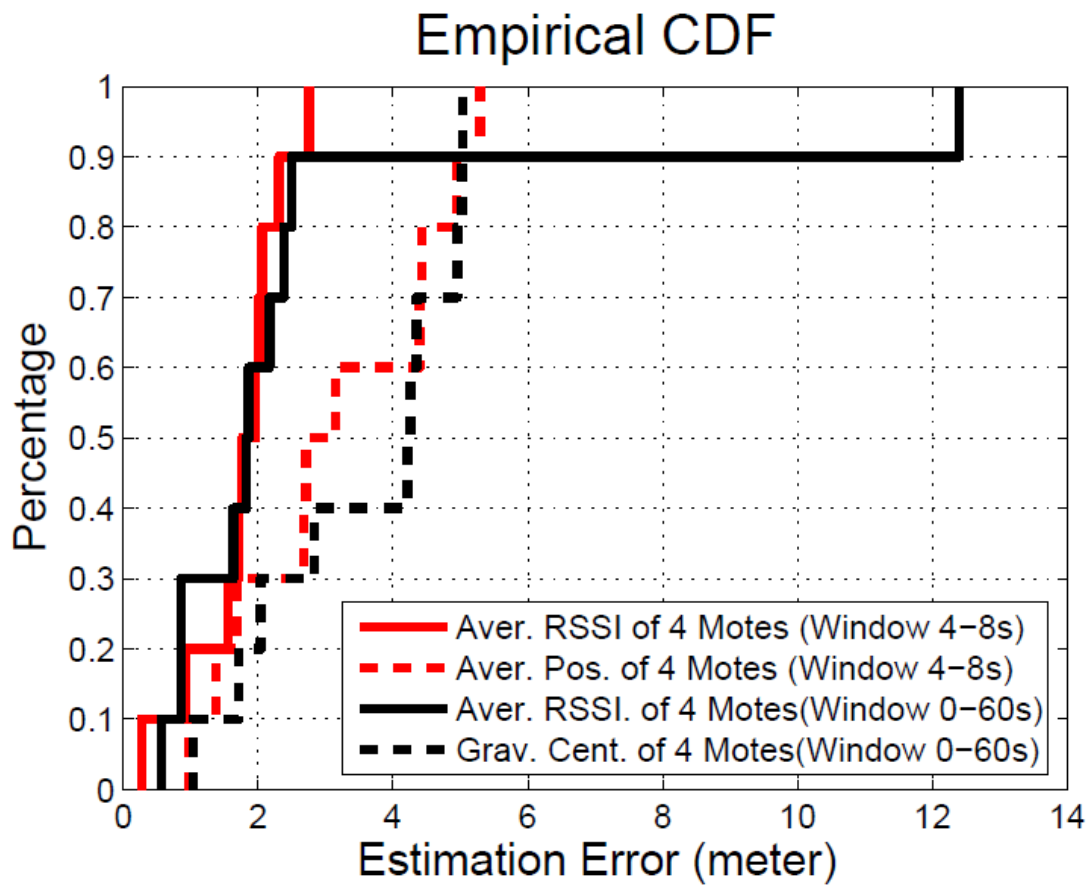

Figure 10. Estimation Error Comparison: 5 Samples Each Target

In general, the estimation of Scenario-B is worse than Scenario-A. This is due to the fact that target positions in Scenario-B are very close to the walls, therefore RSSI measurements are expected to have higher influence from radio reflections and shadowing.

Except for the average RSSI from 4 target motes, the other option is to use the largest RSSI among the four. We use the same setup as used in Fig. 11 to compare the performance of using average RSSI and 
maximum RSSI. The results are plot in Fig. 12 and Fig. 13 for range-free algorithm and range-based algorithm respectively. With range-free algorithm the maximum RSSI performs worse than the average RSSI in both scenarios in most locations. This is due to that we use the same antenna in anchors thus they are not omni-directional. Thus the maximum RSSI does not really show the actual distance between target mote and anchors. With range-based algorithm the difference of estimation error between average RSSI and maximum RSSI is smaller. Only in a few locations we can see a clear gap between curves where maximum RSSI still performs a bit worse than average RSSI. Almost all the estimation errors are below $2 \mathrm{~m}$ except one error with maximum RSSI in Scenario-B is close to $4 \mathrm{~m}$. We can conclude from the experimental results in the two figures that average RSSI performs better than maximum one.

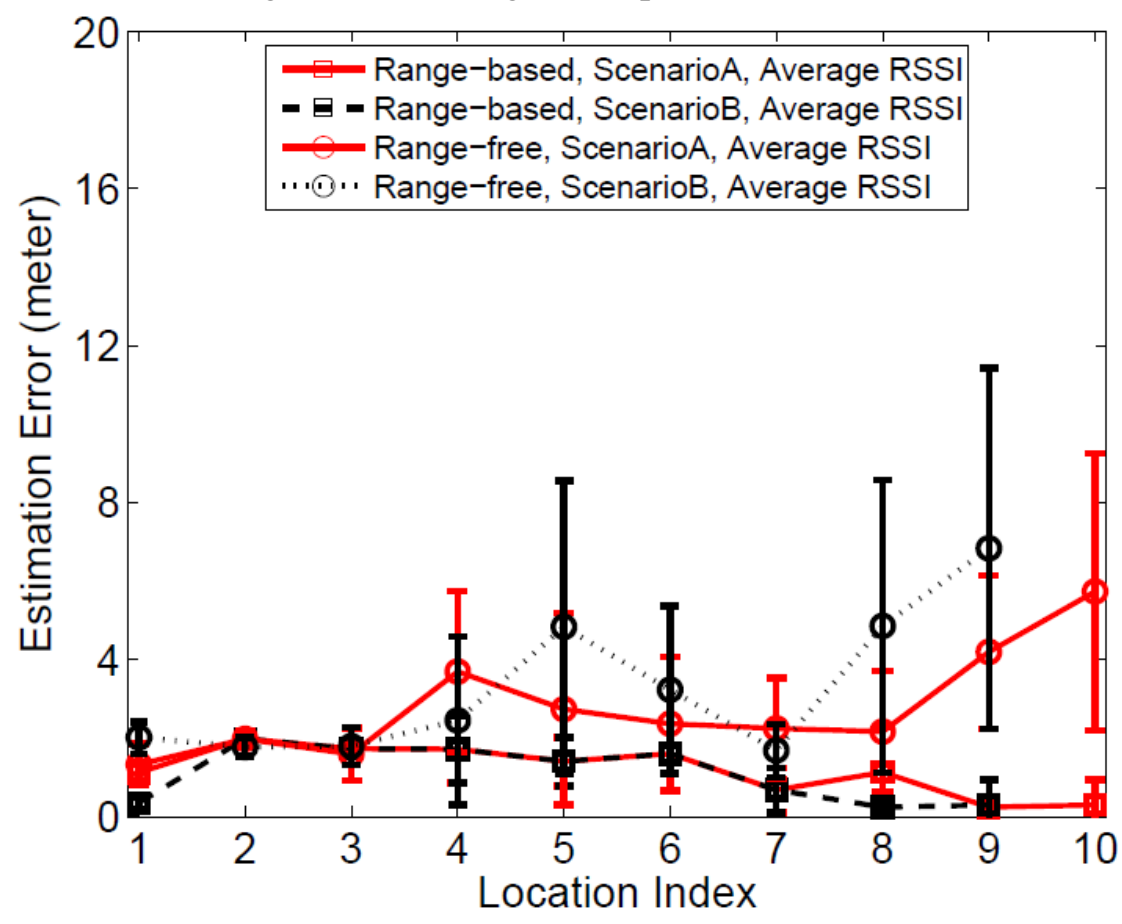

Figure 11. Variation of estimation error on time scale: comparison between range-based and range-free algorithm 


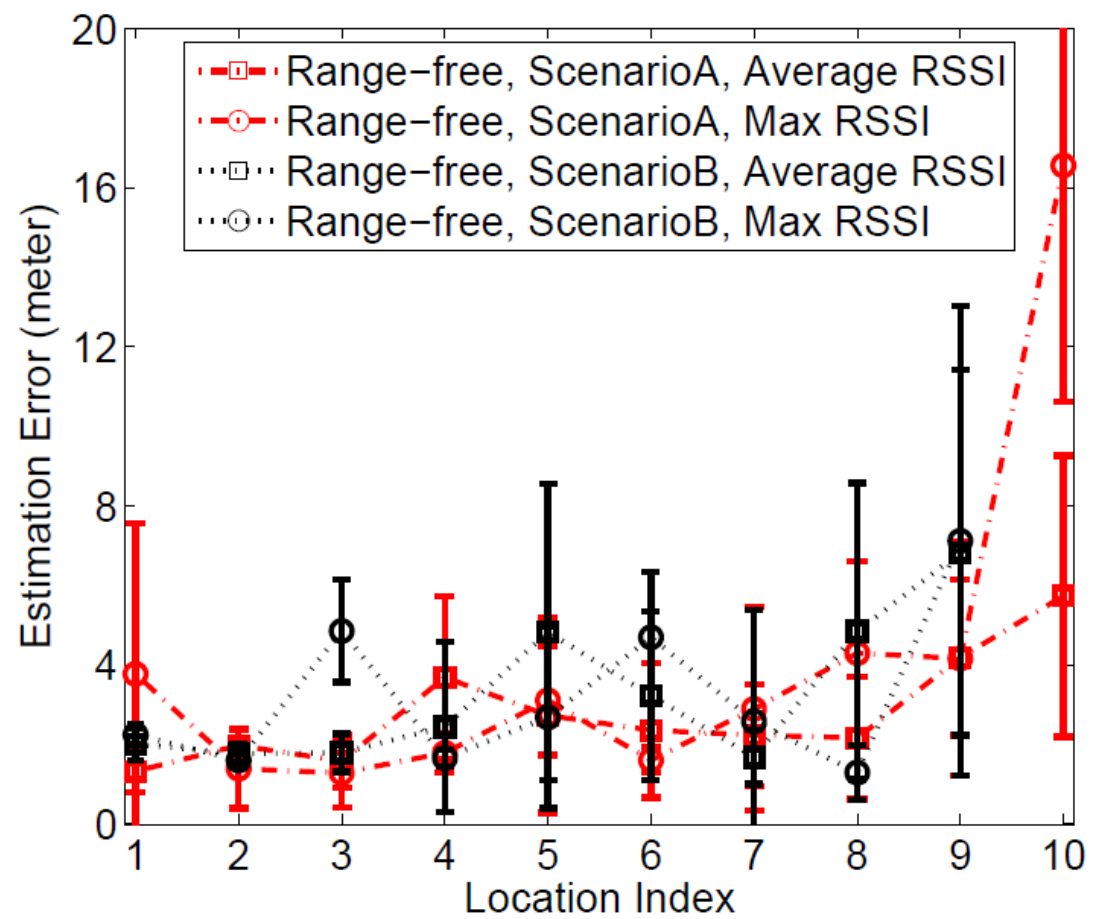

Figure 12. Variation of estimation error on time scale with range-free algorithm

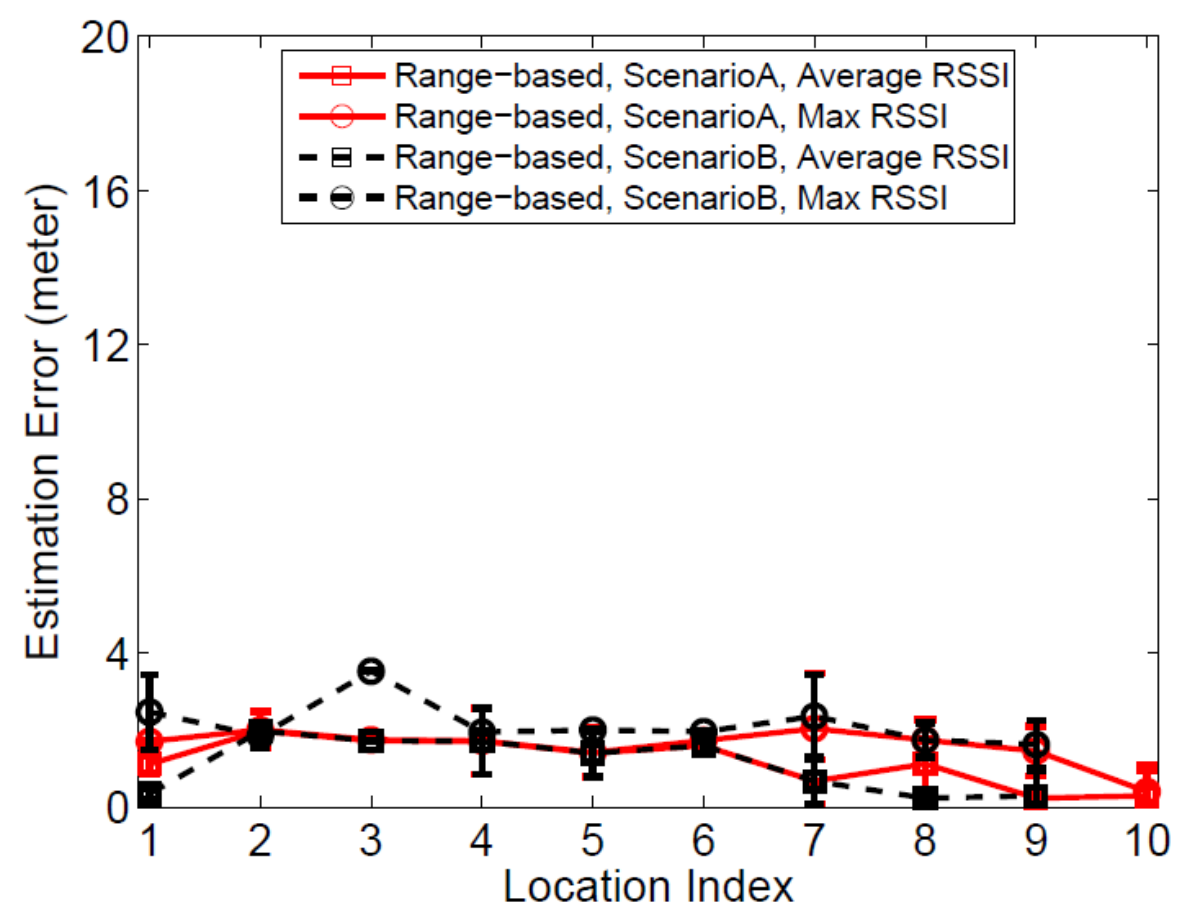

Figure 13. Variation of estimation error on time scale with range-based algorithm

\section{CONCLUSIONS}

In this chapter we first introduced the localization with BASN. Such network provides many novel health monitoring applications for users. In these applications, especially in health care domain, location of the user is essential context information for providing first-aid or to analyze the movement pattern of users. To eliminate the cost of standalone localization we exploit RSSI based localization in of WSNs and 
BASN that is frequently used as the source for localization estimation. Many RSSI-based localization methods are proposed in the literature. One common assumption in these methods is that the devices have omni-directional antennas, which is normally not true in practice. Due to their small size and low cost, the antennas on BASN devices are far from omni-directional. Therefore, we proposed a new method to improve localization accuracy by mitigating the errors caused by deficient antennas and combating fading with spatial diversity. The method uses multiple receivers in a BASN to locate the person who carries them. The method used here has a real application since a person may carry several wireless device in the future. We introduced the method and our range-based and range-free algorithms in this chapter. We investigated the performances of the method with both algorithms through experiments. With the experimental results, we show that the method can improve localization accuracy from a single receiver with both range-based and range-free algorithms. We also show that the accuracy may change over time significantly with range-free algorithm but rather stable with range-based algorithm. The latter algorithm also has a smaller localization error as well. Thus we can recommend with confidence that range-based algorithm works better with the multiple receiver method as long as the empirical curve is also plotted with multiple receivers. The comparison between using maximum and average RSSI suggests that average RSSI works better. Furthermore, average RSSI from a longer duration does not improve the accuracy much.

\section{REFERENCES}

E. Aarts and S. Marzano (2003). The new everyday - views on ambient intelligence. Philips Design.

D. Aguayo, J. Bicket, S. Biswas, G. Judd, and R. Morris (2004). Link level measurements from an 802.11b mesh network. In Proc. ACM SIGCOMM'04, Portland, OR, USA.

X. An, J. Wang, R. V. Prasad, and I. G. M. M. Niemegeers (2006). Opt - online person tracking system for context-awareness in wireless personal network. In Proc. ACM SIGMOBILE REALMAN workshop. Florence, Italy.

P. Bahl and V. N. Padmanabhan (200). Radar: An in-building rf-based user location and tracking system. In Proc. IEEE INFOCOM 2000. Tel-Aviv, Israel.

R. C. Bernhardt (1987). Macroscopic diversity in frequency reuse radio systems. IEEE journal on selected areas in communications, 5(5). 862-870.

CC2420 RF transceiver datasheet. Texas Instruments.(2008). http://focus.ti.com/docs/prod/folders/print/cc2420.html

D.C. Cox, R. Murray, and A.W. Norris (1984). $800 \mathrm{MHz}$ attenuation measured in and around suburban houses. AT\&T Bell Laboratories technical journal. 63(6). 921-954.

P. Deng and P. Z. Fan (2000). An AoA assisted ToA positioning system. In WCC - ICCT 2000: Proceedings of IEEE International Conference on Communication Technology. Beijing, China.

K. Ducatel. et al (2001). Scenarios for ambient intelligence in 2010. In IST Advisory Group (ISTAG), European Commission, Brussels, Belgium,. www.cordis.lu/ist/istag.html

J. Gutierrez, M. Naeve, E. Callaway, M. Bourgeois, V. Mitter, and B. Heile (2001). IEEE 802.15.4: a developing standard for low-power low cost wireless personal area networks. IEEE Network. 15(5). 1219. 
S. Gezici, Z. Tian, G. B. Biannakis, H. Kobayashi, A. F. Molisch, H. V. Poor, Z. Sahinoglu (2005). Localization via ultra-wideband radios. IEEE Signal Processing Magazine. 22(4). 70-84.

T. He, C. Huang, B. M. Blum, J. A. Stankovic, and T. Abdelzaher (2003). Range-free localization schemes for large scale sensor networks. In MobiCom '03: Proceedings of the 9th annual international conference on Mobile computing and networking. New York, NY, USA. 81-95.

J. Hightower and G. Boriello (2001). Location systems for ubiquitous computing. IEEE Computer. 8(5). 57-66.

G. Jin, Xiao-yi Lu, and M. Park (2006). An indoor localization mechanism using active RFID Tag. In SUTC 2006: Proceedings of IEEE International Conference on Sensor Networks, Ubiquitous, and Trustworthy Computing. Taichung, Taiwan.

Erin-Ee-Lin Lau, Boon-Giin Lee and W.-Y. Chung (2008). Enhanced RSSI based high accuracy real-time user location tracking system for indoor and outdoor environments. International Journal on Smart Sensing and Intelligent Systems. 1(2). 534-548.

C. Liu, K. Wu, and T. He (2004). Sensor localization with ring overlapping based on comparison of received signal strength indicator. In Proc. IEEE International Conference on Mobile Ad-hoc and Sensor Systems. Florida, USA.

K. Lorincz and M. Welsh (2005). Motetrack: A robust, decentralized approach to RF-based location tracking. In Proc.International Workshop on Location- and Context-Awareness (LoCA 2005), Oberpfaffenhofen, Germany.

K. Mutukrishnan, M. Lijding, and P. Havinga (2005). Towards smart surroundings: Enabling techniques and technologies for localization. In LoCA 2005: Proceedings of Location-and Context-Awareness: First International Workshop. Oberpfaffenhofen, Germany.

A. Savvides, C.-C. Han, and M. B. Srivastava (2001). Dynamic fine-grained localization in ad-hoc networks of sensors. In Proc. ACM MobiCom 2001. Rome, Italy.

Tmote sky datasheet. Mote IV Corporation (2006). http://www.moteiv.com/products/docs/tmoteskydatasheet.pdf

J. Wang, X. An, R. V. Prasad, and I. Niemegeers (2006). A range-free online person tracking system. In Proc. First International Conference on Pervasive Computing Technologies for Healthcare. Innsbruck, Austria.

K. Yu and I. Oppermann (2004). Performance of UWB position estimation based on time-of-arrival measurements. In UWBST: Proceedings of IEEE Conference on Ultra wideband System and Technology. Kyoto, Japan.

J. Zhao and R. Govindan (2003). Understanding packet delivery performance in dense wireless sensor networks. In Proc. ACM SenSys'03. Los Angeles, CA, USA.

Acknowledgements: This work was supported by Dutch Freeband PNP 2008 project and Dutch IOP GenCom Future Home Networks project. 